\title{
Atomic Chemistry in Turbulent Astrophysical Media
}

\author{
Evan Scannapieco ${ }^{1}$, William J. Gray ${ }^{2}$ and Daniel Kasen ${ }^{3}$ \\ ${ }^{1}$ School of Earth and Space Exploration, Arizona State University, P.O. Box 871404, Tempe, \\ AZ 85287-1494, USA \\ email: evan.scannapieco@asu.edu \\ ${ }^{2}$ Lawrence Livermore National Laboratory, P.O. Box 808, L-038, Livermore, CA 94550, USA \\ email: gray58@llnl.gov \\ ${ }^{3}$ Lawrence Berkeley National Laboratory, Berkeley, CA 94720, USA \\ email: kasen@berkeley.edu
}

\begin{abstract}
We decribe direct numerical simulations of turbulent astrophysical media exposed to the redshift zero metagalactic background. The simulations assume solar composition and explicitly track ionizations, recombinations, and ion-by-ion radiative cooling for hydrogen, helium, carbon, nitrogen, oxygen, neon, sodium, magnesium, silicon, and iron. Each run reaches a global steady state that not only depends on the ionization parameter, $U$, and mass-weighted average temperature, $T_{\mathrm{MW}}$, but also on the the one-dimensional turbulent velocity dispersion, $\sigma_{1 \mathrm{D}}$.

We carry out runs that span a grid of models with $U$ ranging from 0 to $10^{-2}$ and $\sigma_{1 \mathrm{D}}$ ranging from 12 to $58 \mathrm{~km} \mathrm{~s}^{-1}$, and we vary the product of the mean density and the driving scale of the turbulence, $n L$, which determines the average temperature of the medium, from $n L=10^{16}$ to $n L=10^{20} \mathrm{~cm}^{-2}$. The turbulent Mach numbers of our simulations vary from $M \approx 0.5$ for the lowest velocity dispersions cases to $M \approx 20$ for the largest velocity dispersion cases. When $M \lesssim 1$, turbulent effects are minimal, and the species abundances are reasonably described as those of a uniform photoionized medium at a fixed temperature. On the other hand, when $M \gtrsim 1$, dynamical simulations such as the ones carried out here, are required to accurately predict the species abundances.

We gather our results into a set of tables, to allow future redshift zero studies of the intergalactic medium to account for turbulent effects. They are available at http://zofia.sese.asu.edu/ evan/turbspecies/ and will be updated as we increase our parameter study. These results are explained in more detailed in Gray, Scannapieco, \& Kasen (2015), and Gray and Scannapieco (2015)
\end{abstract}

Keywords. Turbulence, ISM: atoms, ISM: abundances

\section{References}

Gray, W. J., Scannapieco, E., \& Kasen, D. 2015, ApJ, 801, 107

Gray, W. J. \& Scannapieco, E. 2015, ApJ, submitted 